\title{
The Impact of the Implementation of Edge Detection Methods on the Accuracy of Automatic Voltage Reading
}

\author{
Kamil Sidor $^{1}$, Anna Szlachta ${ }^{2}$ \\ ${ }^{1}$ Rzeszow University of Technology, al. Powstancow Warszawy 12, 35-959, Rzeszow, Poland \\ ${ }^{2}$ Department of Metrology and Diagnostic Systems, Faculty of Electrical and Computer Engineering, Rzeszow University of \\ Technology, W. Pola street, No.2, 35-959, Rzeszow, Poland
}

\begin{abstract}
The article presents the impact of the edge detection method in the image analysis on the reading accuracy of the measured value. In order to ensure the automatic reading of the measured value by an analog meter, a standard webcam and the LabVIEW programme were applied. NI Vision Development tools were used. The Hough transform was used to detect the indicator. The programme output was compared during the application of several methods of edge detection. Those included: the Prewitt operator, the Roberts cross, the Sobel operator and the Canny edge detector. The image analysis was made for an analog meter indicator with the above-mentioned methods, and the results of that analysis were compared with each other and presented.
\end{abstract}

Keywords: Image analysis, Hough transform, edge detection, LabVIEW environment.

\section{INTRODUCTION}

In everyday life, we have a wide array of skills at our disposal. Among those most important, if not the most important, is the ability to analyse the surrounding world based on the information received through the eyes. The received image is processed by the brain which is a sort of processor processing the image at a very high level. Similar processes take place during a computer image analysis. The main difference consists in the ability to use the full information. Therefore, one may consider the development of machine vision systems as one of the most important tasks of the contemporary computer science. In recent years, vision systems have been gaining an increasing popularity. This results from the fact that they find application in a growing number of areas. They are used in the supervision of industrial processes as well as to control traffic. Processed images are also used in the analysis of satellite photographs of Earth's surface. Computer image analysis allows for obtaining information which otherwise would be impossible to discern with the unaided eye. This allows the detection of lesions in human internal organs and therefore finds application in medicine. Computer techniques of image processing are also used for artistic purposes, including the creation of music videos. Image analysis may also be applicable in metrology, where it may help in taking measurements [1]. An image can be used as a source of information once it is processed in a digital format.

The problem related to determining the position and orientation of a straight line in images concerns many different areas of image processing and computer vision. These may be straight lines in different natural sites, landmarks and complex facilities. Often, they may be identified as various combinations of the linear function.

The detection of a straight line is used, among other things, for finding roads and paths in air photos [2], [3]. In addition to this, detection of a straight line in real time may also be used for finding edges, e.g. for operating a vehicle or a robot [4].

Other applications for line detection are used in the analysis of fingerprints [5]. These applications represent the importance of the problem of line detection in various areas of our lives. Several approaches have been proposed to solve the problem of line detection. The most common solution is the use of the Hough transform.

\section{THE WORK STATION FOR MACHINE READING OF THE MEASURED VALUE ON ANALOG METER}

In the Department of Metrology and Diagnostic Systems of Rzeszow University of Technology, a work station was established to enable machine reading of the measured value on an analog meter [6].

An analog meter does not have any measurement interface and therefore to connect it to the measuring system, PLEOMAX PWC-3900 Pleo Cam II camera was used. The image recorded using the webcam was saved in a graphic file and then processed by the LabVIEW graphic programme environment [7]. The application detects the position of the indicator on the analog meter and defines the 
measurement range on the basis of the clamp analysis. A photograph of the operating system is shown in Fig.1.

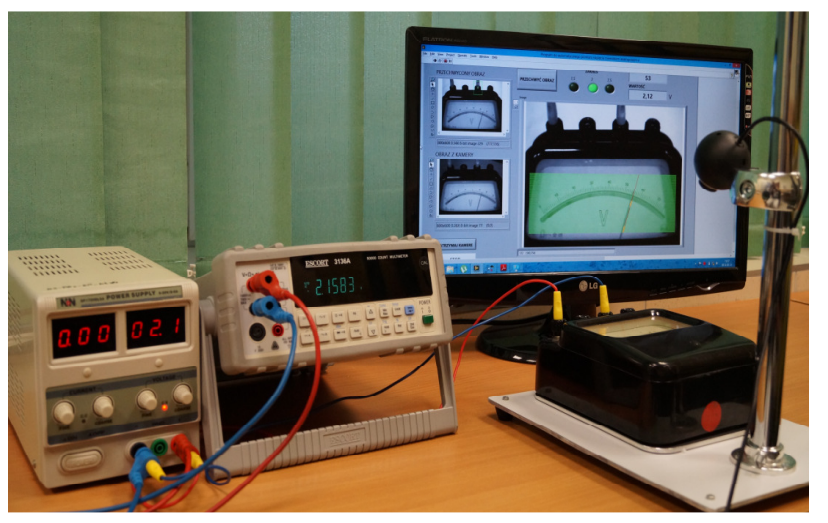

Fig.1. Laboratory work station for the automatic determination of the value measured using an analog meter.

The tool used for research is an analog electromagnetic voltmeter made by Era with the measuring range of: 1,5 V, $3 \mathrm{~V}, 7,5 \mathrm{~V}$. The developed application works with a meter in the abovementioned ranges. The application will operate correctly provided that the webcam is placed directly over the analog meter. To enable the identification of a measuring range, the camera is placed at a constant angle.

The registration programme cooperates with a webcam, and the image from the webcam is stored in the computer memory.

Fig.2. represents a block diagram of the application reading out of the image from the camera connected to the measuring system.

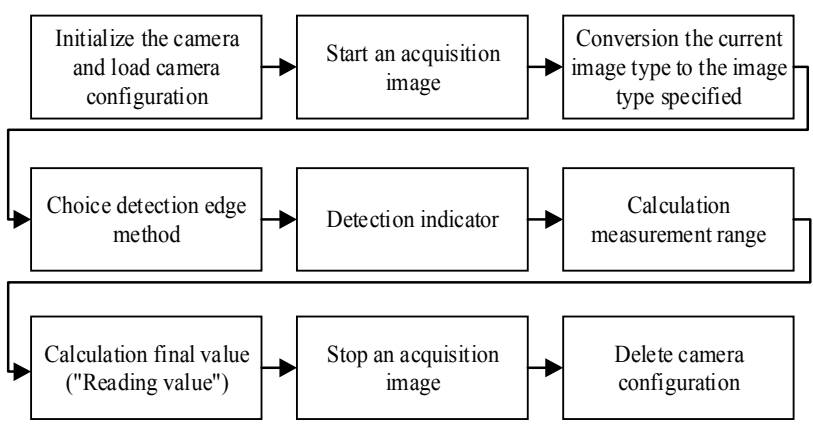

Fig.2. The block diagram of the application.

Fig.3. presents the view of the programme interface window where the correct detection of the indicator occurred.

The highlighted green area determines the part of the image where a straight line was detected corresponding to the indicator coordinates.

The detection of the indicator position in the analysed image is only one of the stages of the determination of the value measured by the analog meter. The detection of the meter's measuring range is equally important. The developed programme assumed that the "+" clamp of the analog meter is connected properly, i.e. it always remains in the same position. In order to specify the measuring range, the programme has to analyse the position of the second clamp.

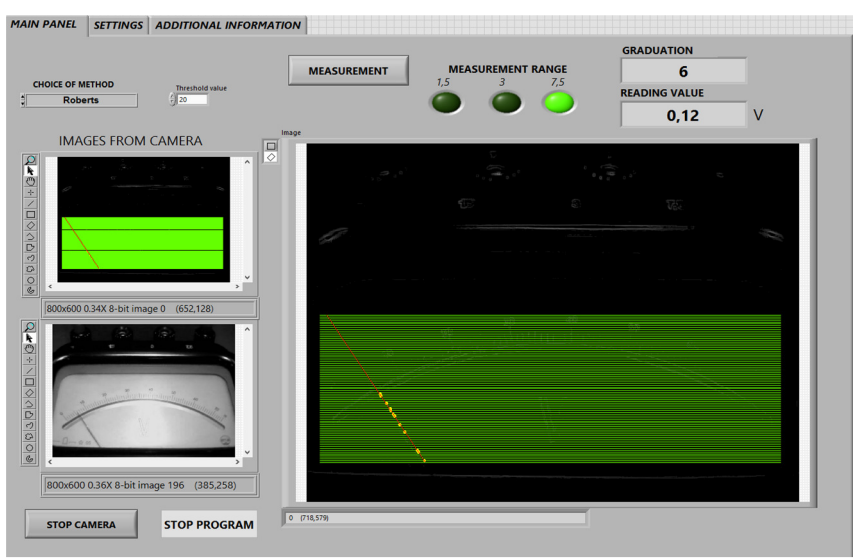

Fig.3. Main page of the application reading the value.

The template matching method was applied, which is used to identify the characteristic features of an image. It consists in comparing the image with a template. The developed algorithm has three templates corresponding to all possible voltmeter clamps. The identification of the measuring range takes place based on the analysis of the $\mathrm{x}$ coordinate of the second consecutive clamp. The comparative method results in a probability function. The method's operation is based on two matrices. The first one is a template matrix. It is a result of saving the template in a digital form. The second matrix is a digital mapping of the analysed image. The search is performed by comparing the values of the template with the values in the image. It is required that the probability measure be defined, because it is rarely the case that the template and image are matched perfectly.

The NI Vision Development tool set includes a special programme for creating patterns called NI Vision Template Editor. It enables the creation of one's own templates and the edition of those created earlier.

\section{HOUGH TRANSFORM}

In order to determine the position of the indicator, the Hough transform [8], [9] was used. A straight line may be represented in the Hough space as an angle of inclination $\theta$ and a distance from the beginning of the coordinate system, described as $\rho$ (Fig.4.).

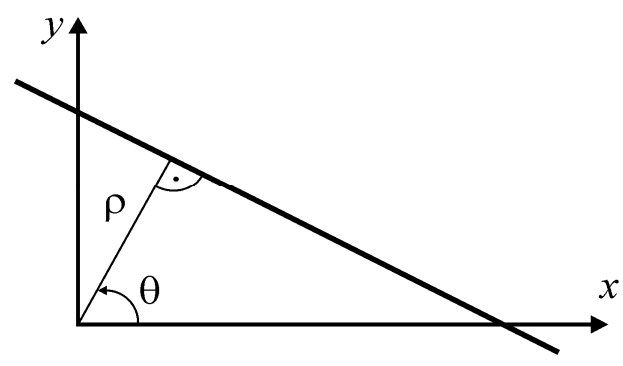

Fig.4. Straight line parameters [10]. 
The straight line determined in this way is described by the formula [10], [11]:

$$
\rho=x \cos (\theta)+y \sin (\theta)
$$

Parameters $\theta$ and $\rho$ make a coordinate system $(\theta, \rho)$ where the straight line is represented as a point (Fig.4.). The coordinate system of this type is called the Hough space [10], [11].

\section{MEthods OF EDGE DETECTION}

To improve the accuracy of the Hough transform, additional methods of edge detection were implemented. The aim of such detection is marking points of a digital image in which the luminance rapidly changes. There are many ways of edge detection, which fall in two categories:

- methods using the analysis of the first-order image derivative (gradient methods) are currently the most popular; they operate by searching the maxima and minima of the first image derivative, and are also called operators or filters;

- methods using the analysis of the second-order derivative in order to detect the edge; usually with the use of zero crossings of the Laplacian or a nonlinear differential expression.

Developed in the LabVIEW environment, the application for edge detection employs only the methods belonging to the former category:

- Roberts cross,

- Sobel operator,

- Prewitt operator,

- Canny edge detector.

They have been applied in the developed application in order to increase the efficiency and accuracy of the reading of a value measured by an analog meter, considering that the indicator is thin and shiny.

\section{A. Roberts cross}

The Roberts cross is one of the simplest operators. It is highly sensitive to local image noise and thus it is not used in the images with a high level of noise due to significant errors in detection. The operating principle is based on the calculation of the differences in luminance of pixels arranged side by side diagonally and then after adding their absolute values. The result of the calculation of differences is expressed as a convolution matrix with $2 \times 2$ kernels. The resulting image of the edge arises after the calculation of module differences from the corresponding elements of the matrix [12]:

$$
g(i, j)=\left\{\begin{array}{l}
0, \text { dla } f(i, j)<T \\
1, \text { dla } f(i, j) \geq T
\end{array} .\right.
$$

\section{B. Sobel operator}

The Sobel operator belongs to the group of simple methods of edge detection. Depending on the factors defining filtering kernels, vertical, horizontal or diagonal edges are detected. The Sobel operator may be expressed as a discrete differentiation operator, computing an approximation of the gradient of the image intensity function. At each point in the image, the operator is the corresponding gradient.

It uses two filters: vertical $\left(S_{y}\right)$ and horizontal $\left(S_{x}\right)$. The $S_{y}$ component determines the gradient value for columns, whereas $S_{x}$ for rows. Kernels are expressed by the equation:

$$
S_{x}=\left[\begin{array}{lll}
-1 & 0 & 1 \\
-2 & 0 & 2 \\
-1 & 0 & 1
\end{array}\right], S_{y}=\left[\begin{array}{rrr}
-1 & -2 & -1 \\
0 & 0 & 0 \\
1 & -2 & -1
\end{array}\right]
$$

Then, based on the formulae, the values of the edges are determined:

$$
\begin{aligned}
G_{\text {mag }} & =\sqrt{\left(S_{x}\right)^{2}+\left(S_{y}\right)^{2}}, \\
G_{d i r} & =\arctan \left(\frac{S_{y}}{S_{x}}\right) .
\end{aligned}
$$

The Sobel operator is much less sensitive to noise than the Roberts cross, but the edges of the obtained image are of different intensity [13].

\section{Prewitt operator}

This operator makes it possible to detect vertical, horizontal and diagonal edges. It enables the calculation of approximations of the derivatives with the use of two $3 \times 3$ kernels which are convolved with the original twodimensional image. The matrices are asymmetrical in relation to the direction of the detected edge. The set of matrices allows to specify the direction from $0^{\circ}$ to $315^{\circ}$.

This method is also known as a formula matching [the kernel] to the actual image. Examples of kernels for angles from $0^{\circ}$ to $45^{\circ}$ are presented in the formula:

$$
P_{x}=\left[\begin{array}{lll}
-1 & 0 & 1 \\
-1 & 0 & 1 \\
-1 & 0 & 1
\end{array}\right], P_{y}=\left[\begin{array}{rrr}
0 & 1 & 1 \\
-1 & 0 & 1 \\
-1 & -1 & 0
\end{array}\right] .
$$

The Prewitt algorithm is a good algorithm for determining the size and the orientation of the edge [14].

\section{Canny's method}

This method was developed in 1986 by John Canny. The Canny edge detector is an edge detection operator that uses a multi-stage algorithm to detect a wide range of edges in images.

The algorithm consists of 4 main stages. The first step involves removing noise with the use of a Gaussian filter. 
As a result, the obtained image is slightly blurred, without any noises.

The next stage features finding the intensity of the image gradient. Considering the fact that edges can be directed in different directions, the algorithm uses four filters to detect the horizontal, vertical, and diagonal edges. Edge operators (e.g. Sobel operator) shall calculate the value of the first derivative of the horizontal and vertical directions. The angle of the edge detection is rounded to four cases representing the horizontal, the vertical and two diagonals (e.g. $\left.0,45,90,135^{\circ}\right)$. The obtained contours are characterised by a certain width and it is not the width of one pixel.

The third step consists in the elimination of non-maximal values of pixel intensity. Pixels constituting the orthogonal cross-section of the contour are analysed, and those lacking maximum intensity values are turned off. The last step includes thresholding aimed at removing unnecessary edges with inclinations below the set threshold.

Canny's algorithm can be used in many different environments. Its parameters can be modified so as to enable the algorithm to recognise edges of various features [15].

\section{EdGE DETECTION IN THE IMAGE}

Fig.5. presents a block diagram representing edge detection with the following methods: the Roberts cross, the Sobel operator and the Prewitt operator.

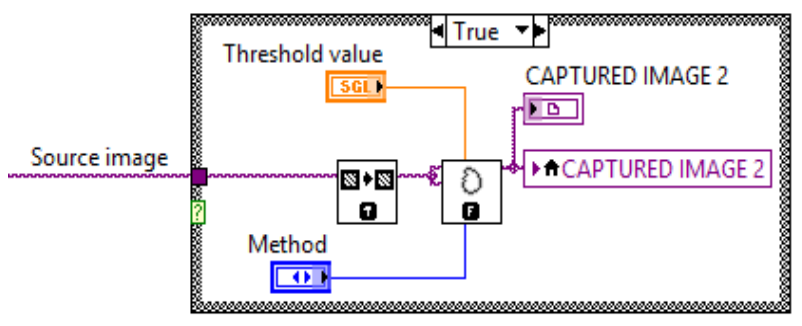

Fig.5. A block diagram representing edge detection with the Roberts cross, the Sobel operator and the Prewitt operator.

For this reason, IMAQ EdgeDetection function was used as it allows the selection of the appropriate method for edge detection [16]. In addition to this, IMAQ EdgeDetection function helps to set thresholding values. It specifies the minimum value of the pixel that should appear on the resulting image. Therefore, it is possible to select edges for display.

Fig.6. presents the list of methods available. The selection is carried out by choosing the desired method from the dropdown menu.

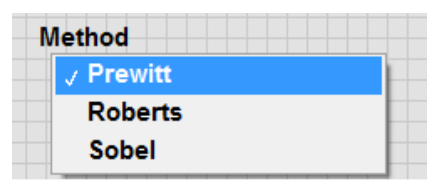

Fig.6. The choice of a method for image edge detection.
Edge detection using Canny's algorithm has been carried out in accordance with the block diagram shown in Fig.7.

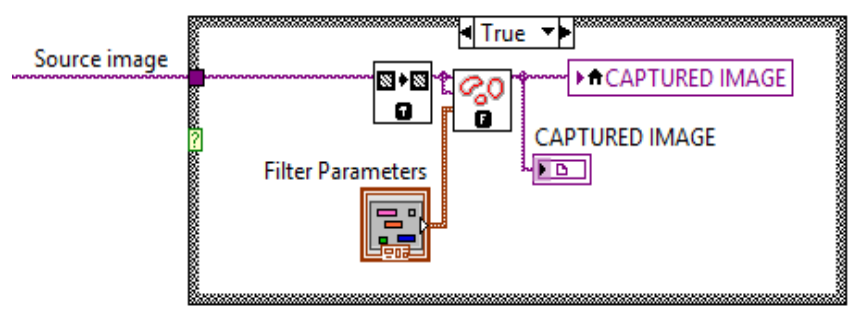

Fig.7. Block diagram describing edge detection using Canny's method.

The main element of the diagram above is the IMAQ Canny Edge Detection VI function which detects edges following Canny's method. There are 4 input parameters to this function. The first one of these is the Sigma parameter. It is the sigma of a Gaussian smoothing filter before edge detection. HThresh (High Threshold) defines the upper percentage of pixel values in the image from which the edge detection algorithm chooses the seed or starting point of an edge segment. It takes values from 0 to 1 . LThres (Low Threshold) is multiplied by the HThresh value to define a lower threshold for all the pixels in an edge segment. The HThresh and LThres parameters determine the upper and lower limit values of thresholding. The last parameter, WindowSize, determines the size of the filter mask of the Gaussian filter. Fig.8. presents the window enabling the specification of the parameters mentioned above.

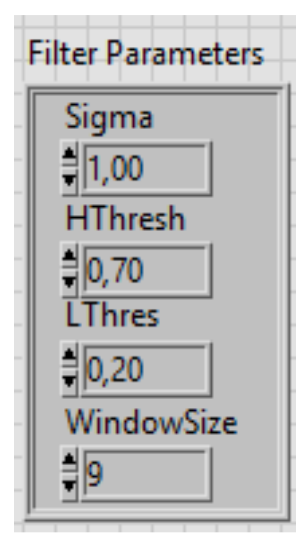

Fig.8. Window showing function parameters in IMAQ Canny Edge Detection [16].

\section{EXPERIMENTAL STUDIES}

During the work with the developed application it has been noticed that the results of the measurements are influenced by the method of edge detection and therefore the application was tested with the use of various edge detection methods.

The voltmeter was divided into 15 voltage values so as to verify the effectiveness of the reading in the broadest possible range of indicator deflection. For each selected voltage value and for each method, automatic measurement 
of voltage value was made based on the analysis of the registered image.

During analysis, each from the chosen methods was used in order to eliminate noise from image threshold. Selected were four threshold values, i.e. 5, 10, 15, 20 (Fig.5.). The experiment was repeated in each measuring point and for each method and for each threshold value. Table 1. shows the results of the studies carried out for the three methods: the Roberts cross, the Sobel operator and the Prewitt operator.

Based on the data presented in Table 1., it can be concluded that the effectiveness of the reading depends on the method used as well as on threshold values for each method.

Table 1. The results of the tests for the Roberts, Sobel and Prewitt methods.

\begin{tabular}{|c|c|c|c|c|c|c|c|c|c|c|c|c|c|}
\hline \multirow{3}{*}{ No. } & \multirow{3}{*}{$\begin{array}{l}\text { User's } \\
\text { reading }\end{array}$} & \multicolumn{12}{|c|}{ Automatic reading with a division into methods and threshold values } \\
\hline & & \multicolumn{4}{|c|}{ Roberts cross } & \multicolumn{4}{|c|}{ Sobel } & \multicolumn{4}{|c|}{ Prewitt } \\
\hline & & 5 & 10 & 15 & 20 & 5 & 10 & 15 & 20 & 5 & 10 & 15 & 20 \\
\hline 1. & 9 & 10 & 10 & 9 & 9 & 10 & 8 & 8 & 9 & 8 & 10 & 10 & 9 \\
\hline 2. & 13 & 12 & 12 & 13 & 13 & 12 & 12 & 13 & 13 & 12 & 13 & 13 & 13 \\
\hline 3. & 18 & 17 & 17 & 18 & 18 & 17 & 17 & 17 & 18 & 17 & 17 & 18 & 18 \\
\hline 4. & 23 & 24 & 24 & 23 & 23 & 23 & 23 & 23 & 23 & 23 & 22 & 23 & 23 \\
\hline 5. & 27 & 28 & 28 & 27 & 27 & 26 & 26 & 26 & 27 & 28 & 26 & 26 & 27 \\
\hline 6. & 33 & 32 & 33 & 33 & 33 & 34 & 33 & 33 & 33 & 34 & 33 & 33 & 33 \\
\hline 7. & 38 & 38 & 38 & 38 & 38 & 38 & 38 & 38 & 38 & 38 & 38 & 38 & 38 \\
\hline 8. & 42 & 42 & 42 & 42 & 42 & 41 & 42 & 42 & 42 & 41 & 42 & 42 & 42 \\
\hline 9. & 47 & 47 & 47 & 47 & 47 & 46 & 47 & 47 & 47 & 46 & 47 & 47 & 47 \\
\hline 10. & 51 & 51 & 51 & 51 & 51 & 51 & 51 & 51 & 51 & 51 & 51 & 51 & 51 \\
\hline 11. & 55 & 55 & 55 & 55 & 55 & 55 & 55 & 55 & 55 & 55 & 55 & 55 & 55 \\
\hline 12. & 60 & 61 & 60 & 60 & 60 & 60 & 60 & 60 & 60 & 60 & 61 & 60 & 60 \\
\hline 13. & 64 & 64 & 64 & 64 & 64 & 64 & 64 & 64 & 64 & 64 & 64 & 64 & 64 \\
\hline 14. & 69 & 69 & 69 & 69 & 69 & 69 & 69 & 69 & 69 & 69 & 69 & 69 & 69 \\
\hline 15. & 73 & 73 & 73 & 73 & 73 & 72 & 72 & 73 & 73 & 73 & 73 & 73 & 73 \\
\hline
\end{tabular}

Table 2. presents the percentage values of the effectiveness of reading values measured by an analog meter. The effectiveness depends to a large extent on the parameter describing the threshold values.

Table 2. A comparison of the reading effectiveness using various methods.

\begin{tabular}{|l|c|c|c|c|}
\hline $\begin{array}{r}\text { Threshold } \\
\text { values }\end{array}$ & $\mathbf{5}$ & $\mathbf{1 0}$ & $\mathbf{1 5}$ & $\mathbf{2 0}$ \\
\hline Roberts cross & $53.3 \%$ & $73.3 \%$ & $100 \%$ & $100 \%$ \\
\hline Sobel operator & $46.7 \%$ & $66.7 \%$ & $80 \%$ & $100 \%$ \\
\hline Prewitt operator & $53.3 \%$ & $66.7 \%$ & $86.7 \%$ & $100 \%$ \\
\hline
\end{tabular}

For the value of 20 , the performance proved to be $100 \%$ for all verified methods. Also for threshold value equalling
15 , the Roberts' method proved to be faultless. Taking into account all tested threshold values, the Roberts cross showed the best performance.

In addition to this, tests using Canny's method (Table 3.) were carried out.

As in the case of the three previous methods, tests were carried out for the same fifteen voltage values. Threshold values (HThresh and LThres) have been selected based on tests. Three ranges of thresholding were proposed, yielding the best results. Table 3. presents the results of the conducted tests.

The research has proved that Canny's method has not achieved the expected results as the results of this method gave the worst reading results of the measured value. Table 4. presents the percentage values of the effectiveness of the reading for Canny's method.

The value range of L95 H95 thresholds proved to be the most effective. The effectiveness of this was merely $60 \%$, which is too low to be considered reliable. Compared with previous methods, Canny's method had fairly low results. 
Table 3. The results of the studies conducted for Canny's method.

\begin{tabular}{|c|c|c|c|c|}
\hline \multirow{2}{*}{ No. } & \multirow{2}{*}{$\begin{array}{l}\text { User's } \\
\text { reading }\end{array}$} & \multicolumn{3}{|c|}{$\begin{array}{c}\text { Thresholding ranges } \\
\text { (L - low threshold, H - high threshold) }\end{array}$} \\
\hline & & L90 H90 & L90 H95 & L95 H95 \\
\hline 1. & 9 & 9 & 8 & 9 \\
\hline 2. & 13 & 12 & 12 & 12 \\
\hline 3. & 18 & 17 & 18 & 18 \\
\hline 4. & 23 & 23 & 23 & 23 \\
\hline 5. & 27 & 27 & 28 & 28 \\
\hline 6. & 33 & 33 & 33 & 33 \\
\hline 7. & 38 & 39 & 39 & 39 \\
\hline 8. & 42 & 43 & 42 & 42 \\
\hline 9. & 47 & 47 & 47 & 48 \\
\hline 10. & 51 & 51 & 52 & 51 \\
\hline 11. & 55 & 55 & 57 & 57 \\
\hline 12. & 60 & 62 & 61 & 61 \\
\hline 13. & 64 & 65 & 64 & 64 \\
\hline 14. & 69 & 69 & 69 & 69 \\
\hline 15. & 73 & 74 & 74 & 73 \\
\hline
\end{tabular}

Table 4. The results of the percentage reading using Canny's method.

\begin{tabular}{|l|l|l|l|}
\hline $\begin{array}{r}\text { Thresholding } \\
\text { Method }\end{array}$ & L90 H90 & L90 H95 & L95 H95 \\
\hline Canny & $53.33 \%$ & $46.67 \%$ & $60.00 \%$ \\
\hline
\end{tabular}

\section{SUMMARY}

The work on the development of systems for image analysis gives increasingly good results and thus is applicable in a growing number of areas. By analysing images, computers take decisions and do not need human control. To a large extent, it facilitates work when computers do some work better than a man. This is the case of image analysis where the computer is able to detect details invisible to the human eye.

The laboratory stand based on the image analysis from camera makes automatic reading of the value measured using an analog meter possible. Application of Hough transform enables to determine the indicator position and in consequence - amount of graduation scale on the analog meter. The calculation of amount of graduation scale into the measured value was executed using the Template Matching method. The template was elaborated which allows to locate the measurement range. One of the factors which provide the correct program operation is appropriate lighting. It was analysed and discussed in the paper [6]. In order to improve the obtained measurement results (i.e. image analysis) the methods of edge detection were used.

The authors used IMAQ Canny Edge Detection function and IMAQ EdgeDetection available in the LabVIEW package.
It was expected that the proposed method of edge detection would increase the effectiveness of the automatic reading of the measured value measured by an analog meter. After carrying out a series of tests, it was concluded that the methods using edge detection gave different results.

The best method has proved to be the Roberts cross, which after setting the correct threshold value gave the most accurate results of reading of the measured value reading, i.e. $100 \%$ effectiveness.

Canny's method has proved to be the least efficient. Despite being the most complex in terms of computing, the method has not proved to be the best. The reason for this may be the width of the edges detected. Too narrow an edge describing an indicator could be incorrectly interpreted by the Hough transform.

\section{REFERENCES}

[1] Tadeusiewicz, R., Korohoda, P. (1997). Komputerowa analiza i przetwarzanie obrazów. Kraków, Poland: Wydawnictwo Fundacji Postępu Telekomunikacji. (in Polish)

[2] Skingley, J., Rye, A.J. (1987). The Hough transform applied to SAR images for thin line detection. Pattern Recognition Letters, 6 (1), 61-67.

[3] Aghajan, H.K., Kailath, T. (1994). SLIDE: Subspacebased line detection. IEEE Transactions on Pattern Analysis and Machine Intelligence, 16 (11), 10571073.

[4] Inigo, R.M., McVey, E.S., Berger, B.J., Wirtz, M.J. (1984). Machine vision applied to vehicle guidance. IEEE Transactions on Pattern Analysis and Machine Intelligence, PAMI-6 (7), 820-826.

[5] Lin, W.C., Dubes, R.C. (1983). A review of ridge counting in dermatoglyphics. Pattern Recognition, 16, $1-8$.

[6] Szlachta, A., Sidor, K. (2014). A laboratory stand for automatic reading of the value measured by an analog meter. Measurement Automation Monitoring, 60 (8), 548-551.

[7] Galabov, K.S. (2012). Application of the NI LabVIEW in the verification of analog measuring instruments. Системи обробки інформичй (Information Processing Systems), 1 (99), 149-151.

[8] Hough, P. (1962). Method and means for recognizing complex patterns, U.S. patent 3069654.

[9] Illingworth, J., Kittler, J. (1998). A survey of the Hough transform. Computer Vision, Graphics, and Image Processing, 44, 87-116.

[10] Duda, R.O., Hart, P.E. (1972). Use of Hough transform to detect curves and lines in pictures. Communications of the ACM, 15 (1), 11-15.

[11] Aggarwal, N., Karl, W.C. (2006). Line detection in images through regularized Hough transform. IEEE Transactions on Image Processing, 15 (3), 582-591.

[12] Sypniewski, G. (2015). Metody wizyjne w automatyzacji spawania. Przeglad Spawalnictwa, 87 (1), 41-49. (in Polish) 
[13] Zawieska, D. (2010). Wybrane operatory detekcji w automatyzacji dopasowywania obrazów cyfrowych bliskiego zasięgu. Archiwum Fotogrametrii, Kartografii i Teledetekcji, 21, 481-491. (in Polish)

[14] Prewitt, J.M.S., Mendelson, M.L. (1966). The analysis of cell images. Annals of the New York Academy of Sciences, 128 (3), 1035-1053.
[15] Watkins, D.C., Sadun, A., Marenka, S. (1995). Nowoczesne metody przetwarzania obrazu. Warszawa, Poland: Wydawnictwo Naukowo - Techniczne. (in Polish)

[16] National Instruments (NI). Image Acquisition and Processing. Manual NI.

Received December 23, 2016. Accepted April 10, 2017. 